\title{
Soluble endoglin and uterine artery Doppler ultrasonography as markers of progression to preeclampsia in women with gestational hypertension: nested case-control study.
}

\author{
Carlos-José Molina-Pérez ${ }^{1}$, Ana Graciela Nolasco-Leaños ${ }^{1}$, Reyes-Ismael Carrillo-Juárez ${ }^{1}$, \\ María Guadalupe Berumen-Lechuga ${ }^{1}$, Irma Isordia-Salas ${ }^{1}$, and Alfredo Leaños-Miranda ${ }^{1}$ \\ ${ }^{1}$ Instituto Mexicano del Seguro Social
}

June 3, 2020

\begin{abstract}
Objective: To determine the clinical usefulness of the soluble endoglin (sEng) and uterine artery Doppler ultrasonography as markers of progression to preeclampsia in women with gestational hypertension (GH). Design: Nested case-control study. Setting: Mexico City, Mexico. Population or sample: 77 singleton pregnant women with GH. Methods: Cases were women who progress to preeclampsia $(n=36)$ and controls were those who did not $(n=41)$. Serum sEng concentrations and uterine artery Doppler ultrasonography were performed at enrollment. Main outcome measures: Progression to preeclampsia and occurrence of adverse outcomes, such as preterm delivery (PD) $<37$ and $<34$ weeks of gestation, small-for-gestational-age (SGA) infant, and fetal growth restriction (FGR). Results: Women with sEng values in the highest tertile had higher risk of progression to preeclampsia, $\mathrm{PD}<34$ weeks of gestation, and FGR, odds ratios (ORs) [?] 3.7. Patients with abnormal uterine artery Doppler pulsatility index ( $>95$ th percentile) had higher risk of progression to preeclampsia, PD $<34$ weeks of gestation, SGA infant, and FGR (ORs [?] 3.3). The presence of notch was associated with higher risk of progression to preeclampsia, PD $<37$ and $<34$ weeks of gestation, SGA infant, and FGR (ORs [?] 2.9). However, logistic regression analysis revealed that only serum sEng was a significant and independent risk factor for progression of GH to preeclampsia, PD <34 weeks of gestation, and FGR (ORs [?]3.1). Conclusions: sEng is a reliable biomarker of progression to preeclampsia, PD and FGR in patients with GH. Compared to sEng, uterine artery Doppler ultrasound has limited clinical usefulness as marker.
\end{abstract}

\section{Hosted file}

Manuscript.docx available at https://authorea.com/users/329254/articles/456533-solubleendoglin-and-uterine-artery-doppler-ultrasonography-as-markers-of-progression-topreeclampsia-in-women-with-gestational-hypertension-nested-case-control-study 

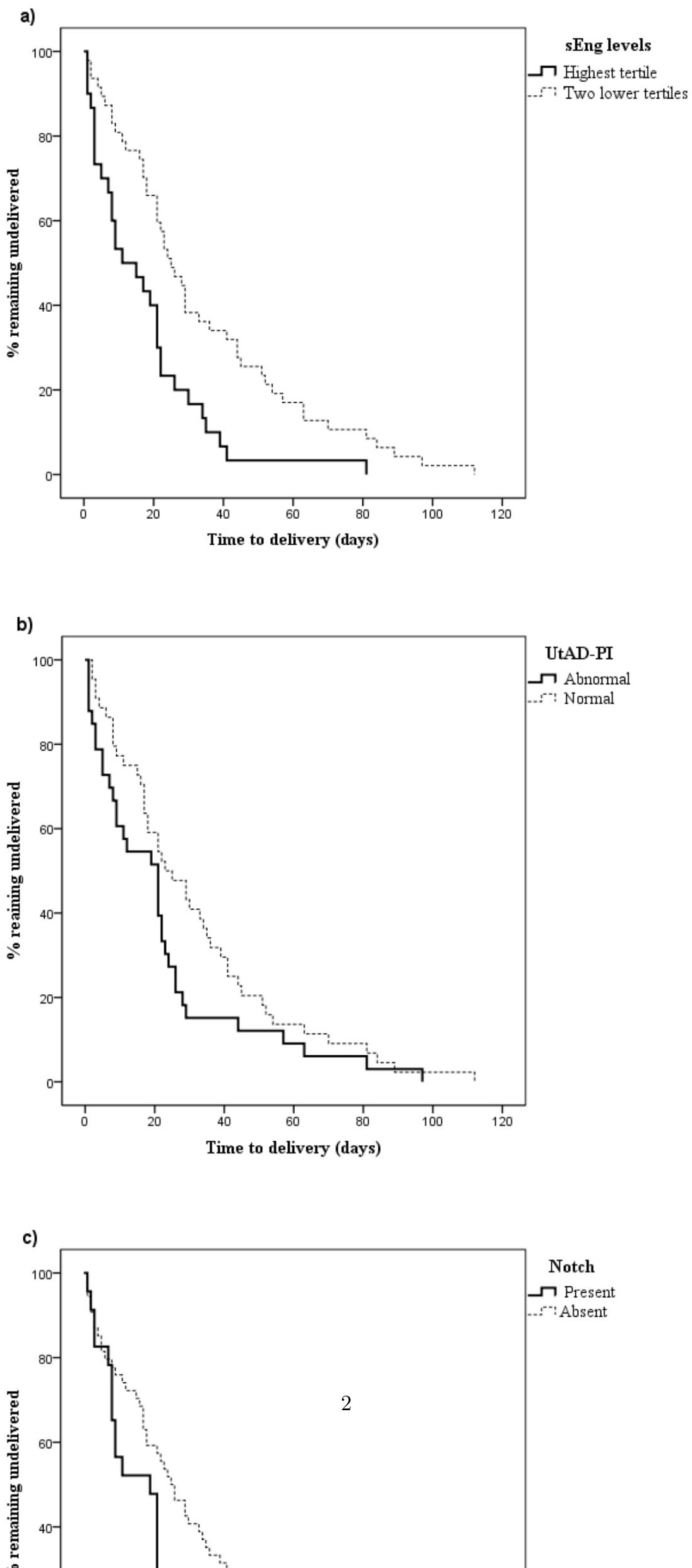